\title{
Integrative multi-omics analysis reveals the \\ landscape of Cyclin-Dependent Kinase (CDK) family genes in pan-cancer
}

Erlin Chen ( $\square$ mncuk169@126.com )

Nantong University

\section{Saiyan Bian}

Nantong University

Wenkai Ni

Nantong University

Peng Wang

Nantong University

\section{Feiran Wang}

Nantong University

\section{Research}

Keywords: Cyclin-dependent kinase, multi-omics, pan-cancer

Posted Date: April 28th, 2020

DOI: https://doi.org/10.21203/rs.3.rs-24985/v1

License: (c) (1) This work is licensed under a Creative Commons Attribution 4.0 International License. Read Full License 


\section{Abstract}

Cyclin-Dependent Kinases (CDKs) get widely involved in cancer development. However, A wealth of conflicting data raise the question whether CDKs serve as oncogenes or cancer suppressors. Here, we integrate multi-omics analysis to explore the core genes of CDK family across multiple cancer types. To our surprise, CDKs are overexpressed in a wide range of cancers, where heterozygous amplification contributed to such transcriptional dysregulation. To explore the functional impact of such overexpression, further pathway analysis revealed that CDK genes cancer hallmarks such as apoptosis and cell cycle. CDK expression associates with 58 drug sensitivity including Trametinib and I-BET-762. Taken together, our study define the landscape of CDKs in a system level and open potential therapeutic opportunities for cancer patients.

\section{Introduction}

Cyclin-dependent kinases (CDKs), a family of serine/threonine kinases, play an important role in the regulation of cell cycle transition (1), consisting of 20 CDKs (CDK1-CDK20) (2). The dysregulation of CDKs in the cell cycle is usually upregulated in various cancers and correlated with cell proliferation, which was endowed with an essential hallmark of cancer (3). Therefore, we believed that dysregulated CDKs have been associated with genesis and development of cancer (4). For example, CDK8 has been identified as an oncogene, and the expression of CDK8 is positively correlated with tumor status, nodal metastasis and stage in breast cancer (5). The high expression level of CDK12 was associated with HER2 status in primary breast cancer, indicating its oncogenic roles in this context (6). Besides, aberrant overexpression of CDK5 could play an oncogenic activity by inducing tumor cell motility in hepatocellular carcinoma and head and neck squamous cell carcinoma $(7,8)$. Therefore, further investigation of CDK family genes is needed to explore the precise role or molecular mechanism underlying the regulation of cancer.

Recent studies have indicated that cancer cells have lots of genomic changes, such as amplification, deletion, translocation, insertion, transversion and copy number variation (CNV) (9). So, it is of great significance to elucidate the relationship between CNV and CDK gene expression for cancer prevention, diagnosis and therapy. Moreover, the initiation and progression of cancers can be viewed as the accumulation of many dysfunctional genes caused by gene mutation and epigenetic modification. DNA methylation as a well-studied epigenetic hallmark that has been determined to cause the abnormal gene expression in human cancers (10), which was associated with genomic instability, such as mutations, may cause chromosomal instability in human cancers (11). Although the linkage between methylation change and chromosomal instability has been widely reported, the direct correlation between CDK gene expression and differential methylation and the frequency of somatic mutation has not been directly estimated in across human cancers.

In this study, we comprehensively integrated multi-omics data to analyze the genetic and methylated alteration of CDK family genes, and explore the regulatory pathways of these genes and the effects of 
gene-related drugs across various human cancers. Our findings will provide new insights into the molecular regulatory mechanisms of CDK family members in cancer occurrence and progression.

\section{Methods}

\section{Datasets}

We downloaded the datasets containing transcriptomic data, CNV, and DNA methylation data of various cancer types from The Cancer Genome Atlas (TCGA) database, such as Thyroid Carcinoma (THCA), Kidney Renal Papillary Cell Carcinoma (KIRP), Liver Hepatocellular Carcinoma (LIHC), Stomach Adenocarcinoma (STAD), Breast Invasive Carcinoma (BRCA), Colon Adenocarcinoma (COAD), Uterine Corpus Endometrial Carcinoma (UCEC), Bladder Urothelial Carcinoma (BLCA), Kidney Renal Clear Cell Carcinoma (KIRC), Kidney Chromophobe (KICH), and Prostate Adenocarcinoma (PRAD).

\section{Dna Methylation Analysis}

We obtained the DNA methylation data from TCGA public methylation profiles produced by the Illumina Human Methylation 450K BeadChip and calculated the Pearson coefficient to achieve a correlation analysis. The FDR was calculated by an unpaired t-test.

\section{Copy Number Variants (cnv) And Somatic Mutation Analysis}

Copy number variations (CNVs) containing heterozygous CNV and homozygous CNV were shown in our TCGA data, and the GISTIC2 method (12) was used to identify CNV segments in various cancers. Pearson coefficient of paired mRNA expression and CNV data was calculated by using the R package. After downloading the somatic mutation data from the TCGA database, we obtained somatic mutations in various cancer types. Then, we analyzed the mutation frequency across various cancer types for estimating the percentage of a specific mutation.

\section{Pathway Analysis}

We obtained the pan-cancer gene expression profile and used clusterProfiler R package to analyze the enriched pathways, including apoptosis, cell cycle, DNA damage response, EMT, PI3K/AKT, RAS/MAPK, RTK, and TSC/mTOR pathways. The threshold p-value $<0.05$ was set as significant enrichment.

\section{Results}




\section{Heterozygous amplification and deletion associates in dysregulation of the CDK family genes in cancers}

RNA sequencing data from the TCGA database were used to analyze the expression levels of CDK family genes in a wide range of cancers. The results showed that most of the CDK family genes, such as CDK1, CDK5, CDK4, CDK2, CDK16, CDK7, CDK6, CDK12, CDK8, CDK17, and CDK13, were up-regulated across various cancer types. In contrast, we also observed that CDK14, CDK15, and CDK20 were down-regulated in some cancer types, and CDK9, CDK11A, and CDK11B remained unchanged (Fig. 1A). To further understand the cause of the core CDK family genes is transcriptionally dysregulated, we studied the CNV in a pan-cancer. The results showed that CDK family genes heterozygous amplification and heterozygous deletion were dominant. CNV analysis of heterozygous amplification and deletion displayed that CDK family genes had the highest amplification due to heterozygosity in KIRP, and have the highest frequency of loss of heterozygosity in $\mathrm{KICH}$ (Fig. 1B). However, the frequencies of homozygous amplification and deletion were very low (Fig. 1C). These above findings suggested that heterozygous amplification and deletion were most likely to result in the transcriptional dysregulation of CDK family genes.

Furthermore, we analyzed the effects of CDK family gene expression on survival risk, which showed that CDK2 and CDK1 survival worse than other genes in KIRC, KIRP, and KICH (Figure S1A). In BLCA, BRCA, KIRC, and STAD, mRNA expression levels of CDK family genes were also shown in Figure S1B. Additionally, gene expression analysis of the CDK family genes in the GTEx dataset revealed that CDK9 has the highest expression value in most normal tissues, such as fallopian tube, nerve, ovary, pituitary, spleen, and uterus, followed by CDK4 and CDK16 (Figure S1C).

\section{Methylated Cdk Family Genes In Cancers}

DNA methylation is an important mechanism involved in aberrant gene expression and carcinogenesis (13). Therefore, we further performed the methylation analysis in various cancer types. CDK6 has the highest increase in methylation rate in BRCA, followed by CDK17 in UCEC and BLCA, and CDK9 in LIHC. The methylation levels of CDK16, CDK1, CDK11B, CDK10, and CDK5 were significantly decreased (Fig. 2A). Previous reports have revealed that the methylation levels may be associated with the regulation of gene expression in cancer tissues (14). Therefore, we applied the correlation analysis between expression and methylation levels of CDK family genes in various cancer types. The results showed that CDK6, CDK9, CDK4, and CDK18 genes with increased methylation levels, and CDK16 and CDK10 genes with decreased methylation levels were negatively correlated with gene expression in some cancer types (Fig. 2B). We also studied the patients' overall survival difference between hypermethylation and hypomethylation, indicating that CDK2, CDK15, CDK10, CDK17, CDK12, CDK11B, and CDK11A survival worse than other CDK members in several cancer types (Figure S3). 


\section{Frequency Analysis Of Cdk Family Genes Mutation In Cancers}

Firstly, we detected the mutation profile in various cancer types and found that most of the CDK family genes were frequently mutated. CDK12 and CDK13 have the highest mutation frequency in UCEC, STAD, BLCA, and COAD (Fig. 3A). An integrated analysis of the mutation types of CDK family genes exhibited that SNPs were dominant (Figure S4A). Missense mutations occur most frequently (Figure S4B). In addition, the mutation has the highest frequency of $\mathrm{C}>\mathrm{T}$ conversion (Figure $\mathrm{S} 4 \mathrm{C}$ ). The median variation of each tumor sample was 1 (Figure S4D). For each mutation type, missense mutation has the highest number of mutations per capita (Figure S4E). The high-frequency mutation CDK family genes were CDK12, CDK13, CDK11B, CDK14, CDK11A, CDK15, CDK19, CDK18, CDK16, and CDK17, respectively (Figure S4F). Moreover, among the variances in the CDK family genes, CDK12 had the highest mutation frequency (28\%), followed by CDK13 (18\%), and CDK11B (10\%) (Fig. 3B).

In addition, CNV pie distribution displayed that heterozygous amplification and heterozygous deletion of CDK family genes were dominant (Figure S2A). We further explored the analysis of the Pearson correlation between CNV and mRNA expression and found that the expression levels of almost all CDK family genes were significantly associated with CNV in BRCA. Among them, CDK12 was the most relevant (Figure S2B).

\section{Pathway Analysis Of Cdk Family Genes}

To further explore the biological functions of these CDK family genes, we performed the correlation pathway analysis. The results showed that CDK activation or inhibition could influence apoptosis, cell cycle, DNA damage response, EMT, PI3K/AKT, RAS/MAPK, RTK, and TSC/mTOR pathways (Figure S5). In these pathways, activation of CDK6, CDK4, CDK2, and CDK1 could activate apoptosis and cell cycle, and inhibit RAS/MAPK. CDK17, CDK15, and CDK14 activation could activate EMT and suppress cell cycle. Besides, activation of CDK16 could activate the cell cycle, and CDK7 could inhibit RAS/MAPK, RTK, and TSC/mTOR pathways (Fig. 4).

\section{Drug Sensitivity Analysis Of Cdk Family Genes}

We detected the drug sensitivity analysis of CDK family genes. The results showed that BX-912, PIK-93, XMD13-2, and KIN001-236 were strongly negatively associated with CDK13 expression (Fig. 5). Z-LLNle$\mathrm{CHO}$ was negatively correlated with CDK6. PLX4720 was negatively associated with CDK2. Moreover, NPK76-囚-72-1 was negatively related to CDK11A. PIK-93 was negatively associated with CDK14. Furthermore, Trametinib was positively associated with CDK19 and CDK1. TGX221 was positively correlated with CDK8. Navitoclax was positively associated with CDK7. In addition, Methotrexate presented the most significant positive correlation with CDK16 expression and had a significant negative 
correlation with CDK9 and CDK11B (Fig. 5). These above candidate small molecule drugs could reverse the expression of CDK family genes, thus providing novel directions and molecular mechanisms for treating cancers.

\section{Discussion}

Cancer is a genetic disease, as occurrence, development, and metastasis are controlled by some genetic and epigenetic alterations in the genome (15). Pan-cancer analysis of multi-omics data, combined with bioinformatics methods, can provide a special platform to identify the common molecular characteristics and the molecular mechanisms of various cancer types (16). In this study, we analyzed the molecular alterations of CDK family genes in multiple aspects, such as the genome, transcriptome, and epigenome. Firstly, gene expression levels of CDK family members were extracted from TCGA Pan-cancer. Across various cancers, we observed that CDK1, CDK5, CDK4, CDK2, CDK16, CDK7, CDK6, CDK12, CDK8, CDK17, and CDK13 were up-regulated in a wide range of cancers, whereas, CDK14, CDK15, and CDK20 were down-regulated in some cancer types, and CDK9, CDK11A, and CDK11B remained unchanged. Moreover, aberrant methylation of many genes has been associated with transcriptional inactivation of genes in various cancers (17). To determine the correlation between methylation and expression, we screened tumors with different levels of methylation and analyzed the correlation between methylation and target gene expression in different types of cancers. We found that the methylation levels of CDK4, CDK6, and CDK18 genes were significantly associated with gene expression in some cancer types. Previous evidence demonstrated that CDK4 and CDK6 could be frequently considered together as promoters of G1 progression (18). As a new regulator of genome stability, CDK18 could prevent DNA damage accumulation and genome instability (19).

Due to the deregulation of cell cycle across a broad range of cancers, cancer cells can frequently show aberrant proliferation, genomic instability consisting of increased DNA mutations and chromosomal aberrations, as well as chromosomal instability (1). In the present study, we identified the genetic mutations in the frequently mutated genes of the CDK family in different cancers. The most mutation frequency was CDK12, followed by CDK13. In line with the above, CDK12 mutations have also been reported in some cancer types, such as lung cancer (20) lymphoma (21), and advanced carcinoma of unknown primary (22). Notably, it is well known that CDK12 and CDK13 have similar biological processes, with both regulating RNA splicing and alternative splicing, maintaining self-renewal in embryonic stem cells (23). Collectively, these findings indicated that multilevel data integration exhibited CDK members with epigenetic phenotypes and distinct mutations.

To understand the functional relevance of CDK family genes, we further assessed their involved signaling pathways. We showed that activation of CDK6, CDK4, CDK2, and CDK1 could activate apoptosis and cell cycle, and inhibit RAS/MAPK. CDK17, CDK15, and CDK14 activation could activate EMT and suppress cell cycle. Besides, activation of CDK16 could activate the cell cycle, and CDK7 could inhibit RAS/MAPK, RTK, and TSC/mTOR pathways. Since cell cycle abnormalities are common in different types of cancer, it has always been considered a potential therapeutic target (24). Thus, it is of great significance to 
elucidate the functional roles of these CDK genes as biomarkers for therapeutic intervention. In addition, due to their role in cell cycle control, CDKs are viewed as targets of genetic manipulations in various cancers, leading to accelerate the development of small molecule drugs against CDKs as an anticancer approach (25). Therefore, we screened the CDK family genes with small molecule drugs to find potential candidate drugs that could reverse abnormally expressed CDK genes in various cancer tissues. Our analysis revealed that BX-912, PIK-93, XMD13-2, and KIN001-236 were negatively associated with CDK13 expression. Z-LLNle-CHO was negatively correlated with CDK6. PLX4720 was negatively associated with CDK2. Besides, NPK76-X-72-1 was negatively related to CDK11A. PIK-93 was negatively associated with CDK14. Trametinib was positively associated with CDK19 and CDK1. TGX221 was positively correlated with CDK8. Navitoclax was positively associated with CDK7. Furthermore, Methotrexate presents the most significant positive correlation with CDK16 expression and has a significant negative correlation with CDK9 and CDK11B. Previous studies have demonstrated that P276-00, as an inhibitor of CDK1, CDK4, and CDK9, could make pancreatic cancer cells sensitive to gemcitabine-induced apoptosis, and inhibit tumor growth and angiogenesis (26). Flavopiridol could also act as a pan-CDK-inhibitor of CDK1, $2,4,6,7$ and 9 (27), and the application of this drug in the treatment of chronic lymphocytic leukemia has achieved satisfactory results (28). Likely, these drugs of our research results could act as chemotherapeutic agents and be widely used to improve the effects of cancer therapeutics.

In conclusion, this study has demonstrated the differential expression of CDK family genes and revealed the cause of abnormal gene expression. We also found that CDK genes associates with cancer hallmarks such as RAS/MAPK and apoptosis. Further drug resistance analysis showed that CDK globally impacts drug effectiveness including Trametinib and Methotrexate. These findings provide new insights into carcinogenesis and unravel new mechanisms of CDK genes that may be further investigated in the future.

\section{Declarations}

\section{Competing interests statement}

The authors confirm that there are no conflicts of interest.

\section{Ethics approval and consent to participate}

Not required.

\section{Consent for publication}

All authors approved.

\section{Availability of data and material}

All data are available on request. 


\section{Funding}

None.

\section{Author contribution}

Conceptualization, Erlin Chen; Data curation, Erlin Chen, Saiyan Bian, Wenkai Ni; Formal analysis, Erlin Chen, Saiyan Bian, Wenkai Ni; Methodology, Erlin Chen; Project administration, Erlin Chen, Peng Wang; Resources, Erlin Chen, Peng Wang; Supervision, Erlin Chen, Peng Wang; Validation, Erlin Chen, Peng Wang; Visualization, Erlin Chen, Peng Wang; Writing - original draft, Erlin Chen, Feiran Wang; Writing review \& editing, Erlin Chen, Feiran Wang, Peng Wang.

\section{Acknowledgements}

None.

\section{References}

1. Malumbres M, Barbacid M. Cell cycle, CDKs and cancer: a changing paradigm. Nature Reviews Cancer;9(3):153-66.

2. Malumbres M, Harlow E, Hunt T, Hunter T, Lahti JM, Manning G, et al. Cyclin-dependent kinases: a family portrait.11(11):1275-6.

3. Hanahan D, Weinberg RA. Hallmarks of Cancer: The Next Generation. Cell 2011;144(5):646-74.

4. Garciareyes B, Kretz A, Ruff J, Von Karstedt S, Hillenbrand A, Knippschild U, et al. The Emerging Role of Cyclin-Dependent Kinases (CDKs) in Pancreatic Ductal Adenocarcinoma. International Journal of Molecular Sciences 2018;19(10):3219.

5. Xu D, Li C, Zhang X, Gong Z, Chan CH, Lee SW, et al. Skp2-MacroH2A1-CDK8 axis orchestrates G2/M transition and tumorigenesis. Nat Commun 2015;6(1):6641-.

6. Naidoo K, Wai PT, Maguire SL, Daley F, Natrajan R. Evaluation of CDK12 Protein Expression as a Potential Novel Biomarker for DNA Damage Response Targeted Therapies in Breast Cancer. Molecular Cancer Therapeutics 2017;17(1).

7. Targeting STAT3/miR-21 axis inhibits epithelial-mesenchymal transition via regulating CDK5 in head and neck squamous cell carcinoma. Molecular Cancer;14(1):213.

8. Targeting cyclin dependent kinase 5 in hepatocellular carcinoma - A novel therapeutic approach. Journal of Hepatology;63(1):102-13.

9. Kumar R, Patiyal S, Kumar V, Nagpal G, Raghava GPS. In Silico Analysis of Gene Expression Change Associated with Copy Number of Enhancers in Pancreatic Adenocarcinoma. International Journal of Molecular Sciences 2019;20(14):3582.

10. Lienert F, Wirbelauer C, Som I, Dean A, Mohn F, Schübeler D. Identification of genetic elements that autonomously determine DNA methylation states. Nature Genetics;43(11):1091-7. 
11. Xu GL, Bestor TH, Bourc'his b, Hsieh CL, Viegas-P E, Iquignot. Chromosome instability and immunodeficiency syndrome caused by mutations in a DNA methyltransferase gene. Nature 1999;402(6758):187-91.

12. Sack LM, Davoli T, Li MZ, Li Y, Xu Q, Naxerova K, et al. Profound Tissue Specificity in Proliferation Control Underlies Cancer Drivers and Aneuploidy Patterns. Cell 2018;173(2):499-514.

13. Goodman Jl, Watson RE. Altered DNA methylation: a secondary mechanism involved in carcinogenesis. Annual Review of Pharmacology and Toxicology 2002;42(1):501-25.

14. Baylin SB, Esteller M, Rountree MR, Bachman KE, Herman JG. Aberrant patterns of DNA methylation, chromatin formation and gene expression in cancer. Human Molecular Genetics 2001;10(7):687-92.

15. Wong MCS, Jiang JY, Liang M, Fang Y, Yeung MS, Sung JJY. Global temporal patterns of pancreatic cancer and association with socioeconomic development. Scientific Reports;7(1):3165.

16. Hasin Y, Seldin M, Lusis A. Multi-omics Approaches to Disease. Genome Biology 2017;18(1).

17. Patel N, Black J, Chen X, Marcondes AM, Grady WM, Lawlor ER, et al. DNA Methylation and Gene Expression Profiling of Ewing Sarcoma Primary Tumors Reveal Genes That Are Potential Targets of Epigenetic Inactivation. 2012;2012:498472.

18. Wood DJ, Endicott JA. Structural insights into the functional diversity of the CDK-cyclin family. Open Biology 2018;8(9):180112.

19. Giancarlo B, Staples CJ, Anil G, Patterson KW, Bryne DP, Myers KN, et al. Human CDK18 promotes replication stress signaling and genome stability. Nucleic Acids Research 2016(18):18.

20. Zhang K, Zhang M, Zhu J, Hong W. Screening of gene mutations associated with bone metastasis in nonsmall cell lung cancer. Journal of Cancer Research \& Therapeutics 2016;12(7):186.

21. Julia, Turbiner, Geyer, Shivakumar, Subramaniyam, Yanwen, et al. Lymphoblastic transformation of follicular lymphoma: a clinicopathologic and molecular analysis of 7 patients.

22. Subbiah IM, Tsimberidou A, Subbiah V, Janku F, Roy-Chowdhuri S, Hong DS. Next generation sequencing of carcinoma of unknown primary reveals novel combinatorial strategies in a heterogeneous mutational landscape. 2017;4(5-6):47-56.

23. Dai Q, Lei T, Zhao C, Zhong J, Tang Y-z, Chen B, et al. Cyclin K-containing Kinase Complexes Maintain Self-renewal in Murine Embryonic Stem Cells. Journal of Biological Chemistry;287(30):25344-52.

24. None. The Cell Cycle. American Biology Teacher;76(7):478-9.

25. Dickson MA, Schwartz GK. Development of Cell Cycle Inhibitors for Cancer Therapy. Current Oncology 2009;16(2):36-43.

26. Subramaniam D, Periyasamy G, Ponnurangam S, Chakrabarti D, Sugumar A, Padigaru M, et al. CDK-4 Inhibitor P276 Sensitizes Pancreatic Cancer Cells to Gemcitabine-Induced Apoptosis. Molecular Cancer Therapeutics;11(7):1598-608.

27. Asghar U, Witkiewicz AK, Turner NC, Knudsen ES. The history and future of targeting cyclindependent kinases in cancer therapy. Nature Reviews Drug Discovery 2015. 
28. Awan FT, Jones JA, Maddocks K, Poi M, Grever MR, Johnson A, et al. A phase 1 clinical trial of flavopiridol consolidation in chronic lymphocytic leukemia patients following chemoimmunotherapy. Annals of Hematology;95(7):1137-43.

\section{Supplementary Figure Legends}

Figure S1. CDK genes are transcriptionally dysfunctional in cancers. (A) The prognostic value of CDK genes. (B) CDK gene expression differs in cancer subtypes. (C) The expression of CDK genes in normal samples.

Figure S2. Copy number variation impacts CDK gene expression. (A) The copy number variation subtypes of CDK genes across cancer. (B) Correlation between Copy number variation and gene expression. Hete Amp: heterozygous amplification; Hete Del: heterozygous deletion; Homo Amp: homozygous amplification; Homo Del: homozygous deletion; None: no CNV

Figure S3. The prognostic value of CDK gene methylation across cancer types.

Figure S4. The summary of CDK gene variants in human cancers. (A) Variant type, (B) Variant classification, (C) Single nucleotide variation class, (D) Number of variants per sample, (E) Variant classification summary, and (F) Frequency of mutation of CDK genes across cancer types.

Figure S5. Percentage (number of activate or inhibit cancer types) of the gene's function (activation or inhibition) for each pathway across various cancers.

\section{Figures}




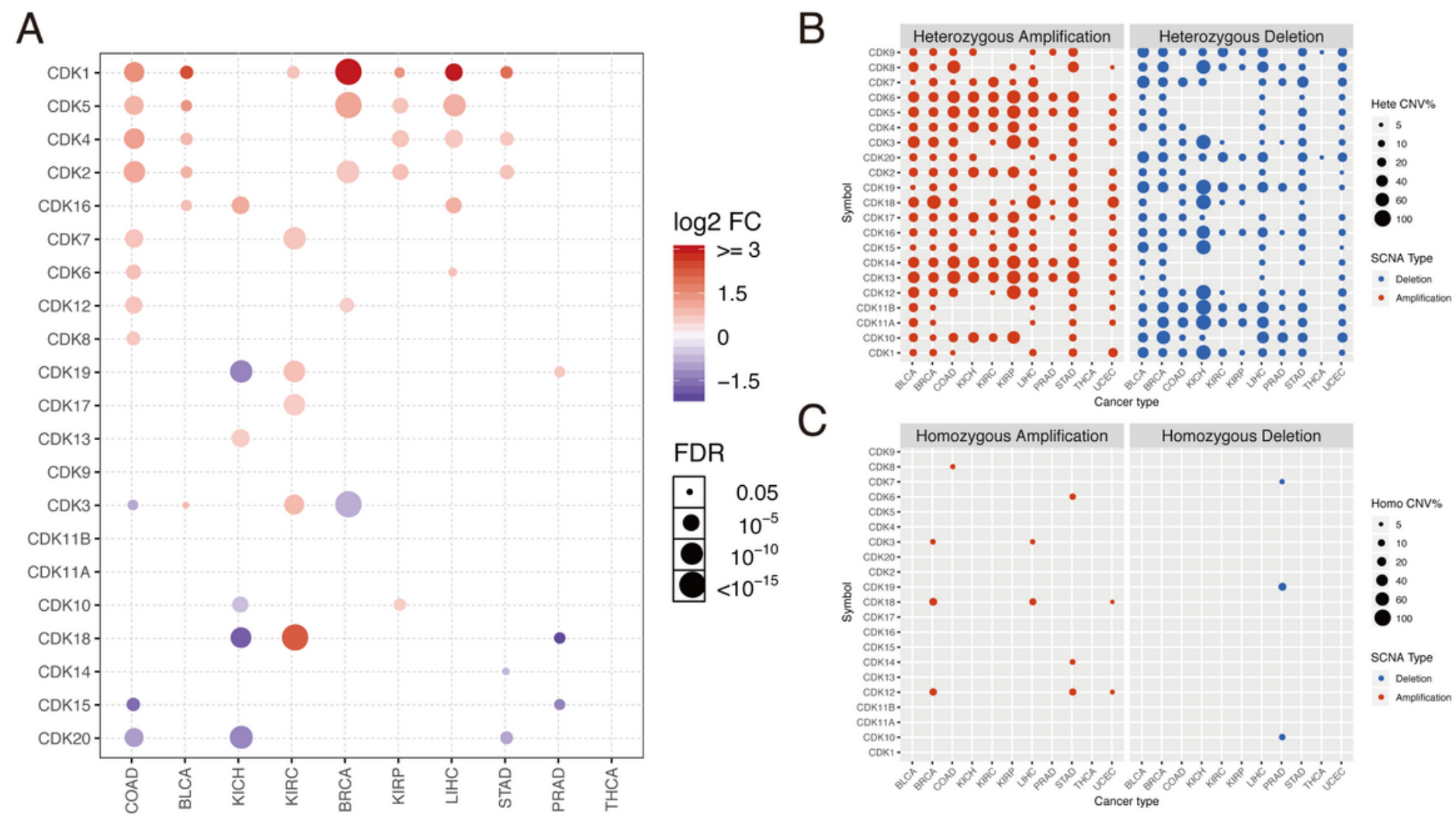

Figure 1

CDK family genes are widely overexpressed across human cancers. (A) CDK genes are transcriptionally up-regulated in a broad spectrum of cancers. Copy number variations of CDK genes including (B) Heterozygous amplification and (C) Homozygous Amplification are common. 


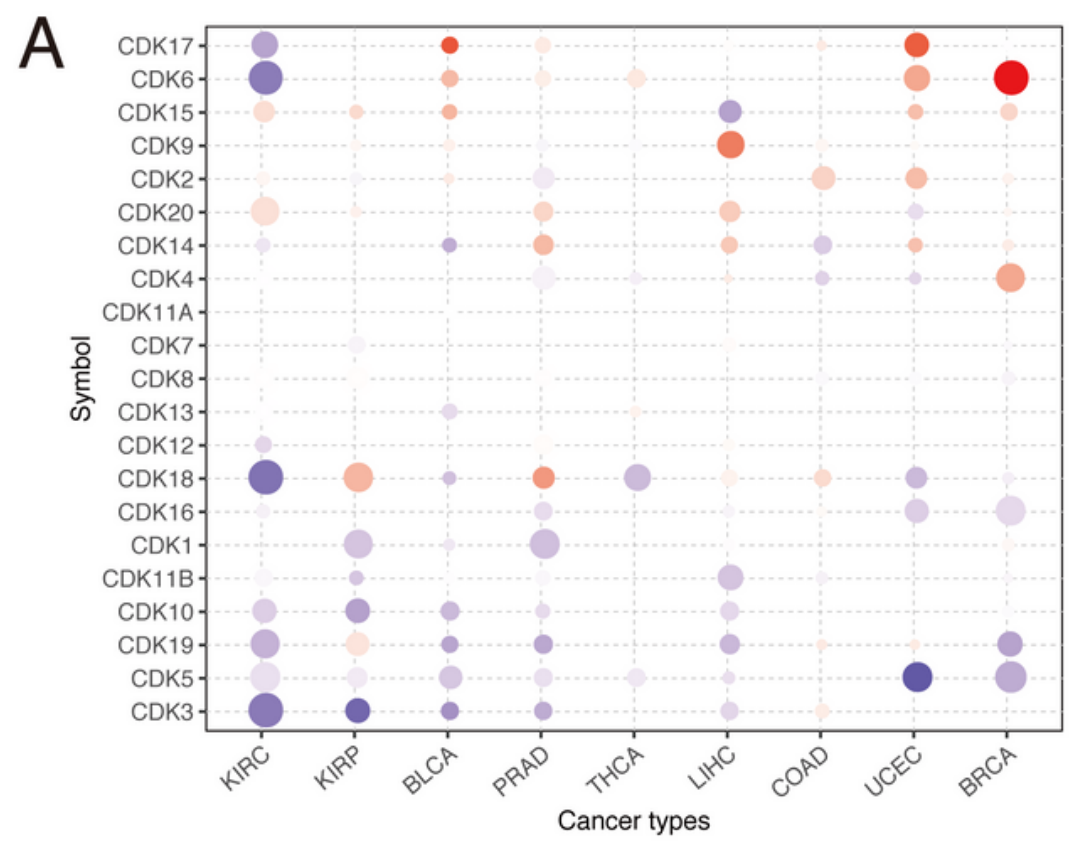

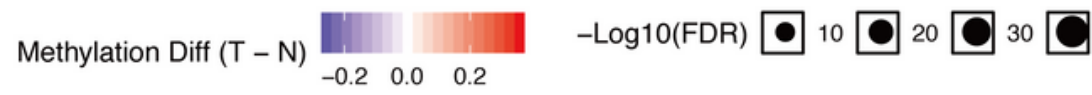

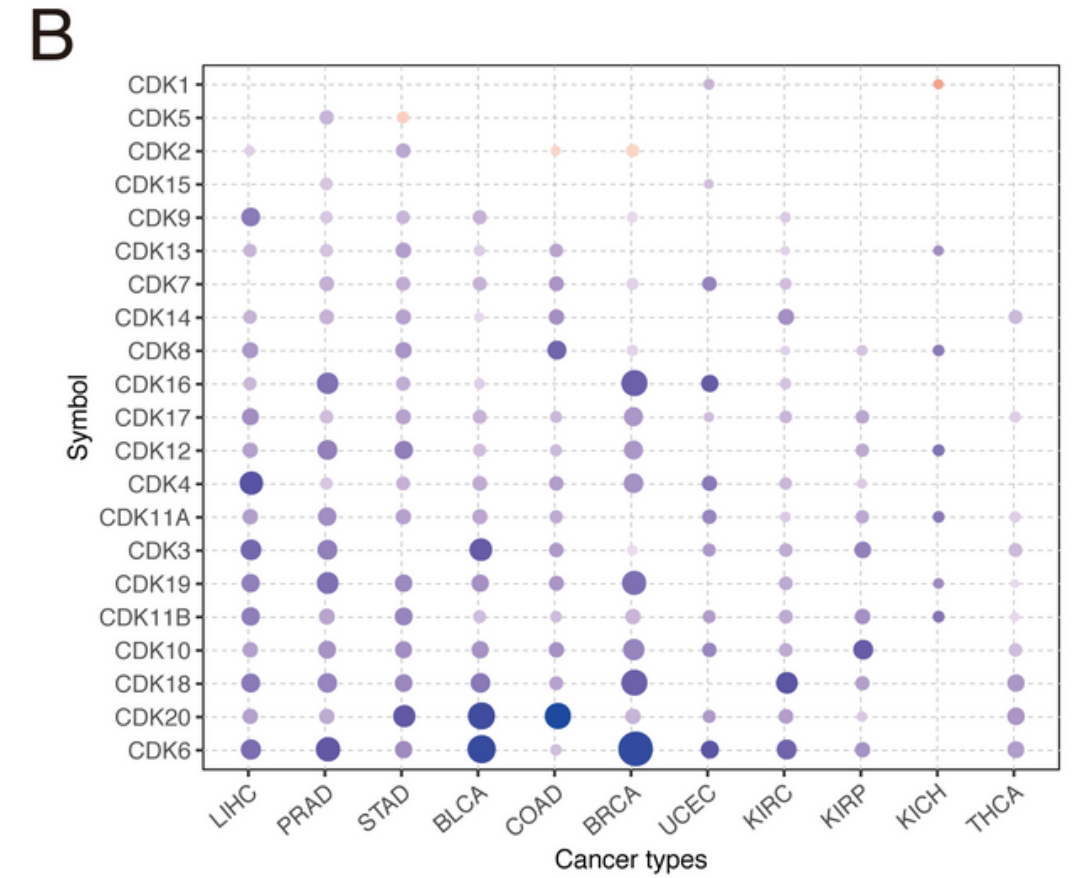

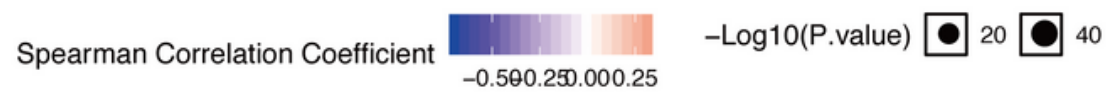

\section{Figure 2}

CDK family is epigenetically de-methylated. (A) The differential methylation profile of CDK genes in human cancers. (B) Correlation between methylation and expression of CDK genes. 
A

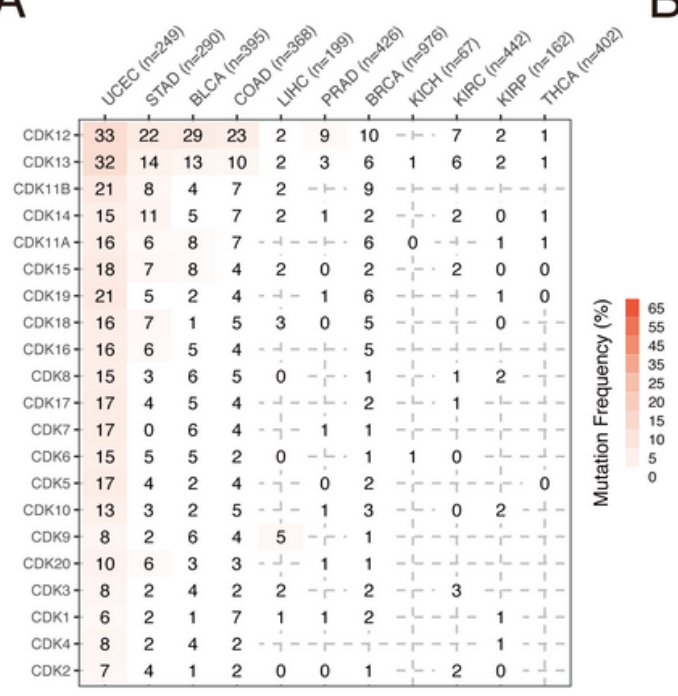

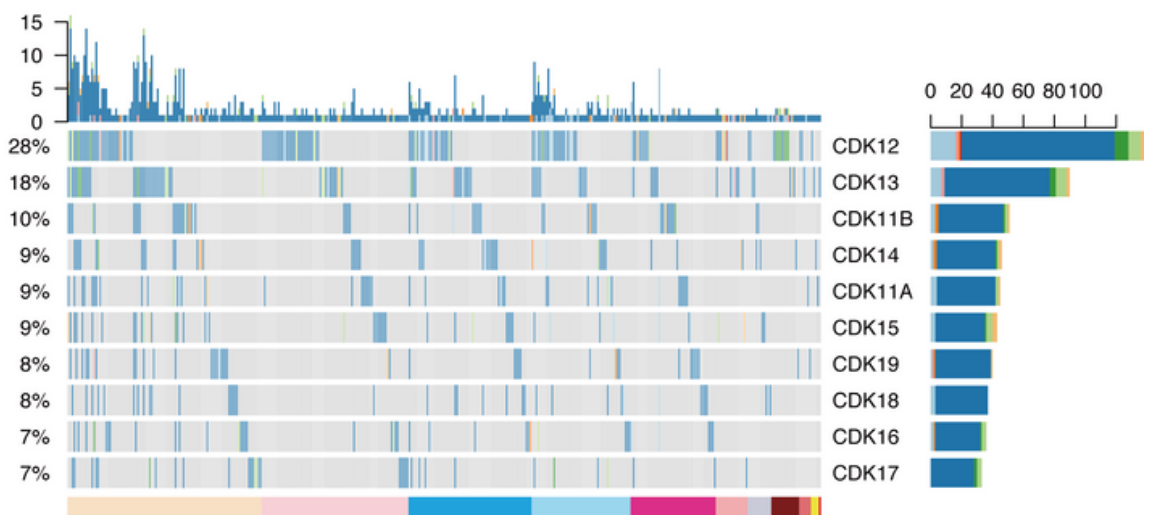

Cancer_Types Nonsense_Mutation Multi_Hit BRCA

THCA COAD LIHC STAD UCEC

BLCA

$\mathrm{KICH}$

KIRC

KIRP

PRAD
[ Missense_Mutation $\quad$ Frame_Shift_Ins

- Splice_Site

In_Frame_Del

\section{Figure 3}

Mutation also contributes to the dysfunctional CDK family in cancers. (A) The Mutation frequency of CDK genes. (B) A summary plot of variants in each sample. 


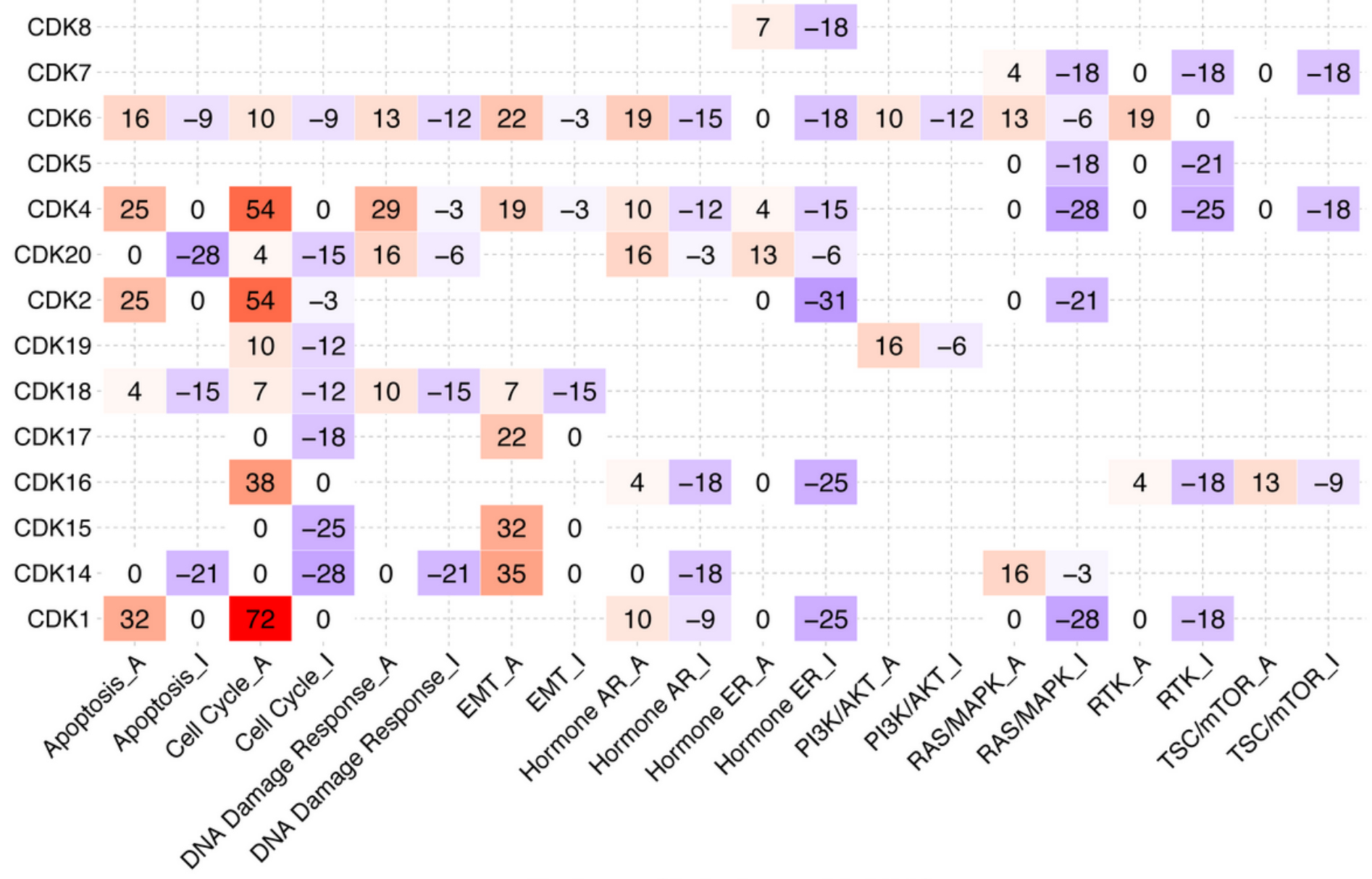

Pathway (A:Activate; I:Inhibit)

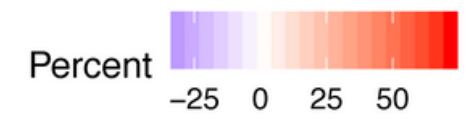

\section{Figure 4}

CDK genes widely associate with hallmark cancer pathways in pan-cancer. Heatmap of genes that have a function (inhibit or activate) in at least 5 cancer types. Pathway_a represents activation of this pathway, inhibition in a similar way showed as pathway_i. 


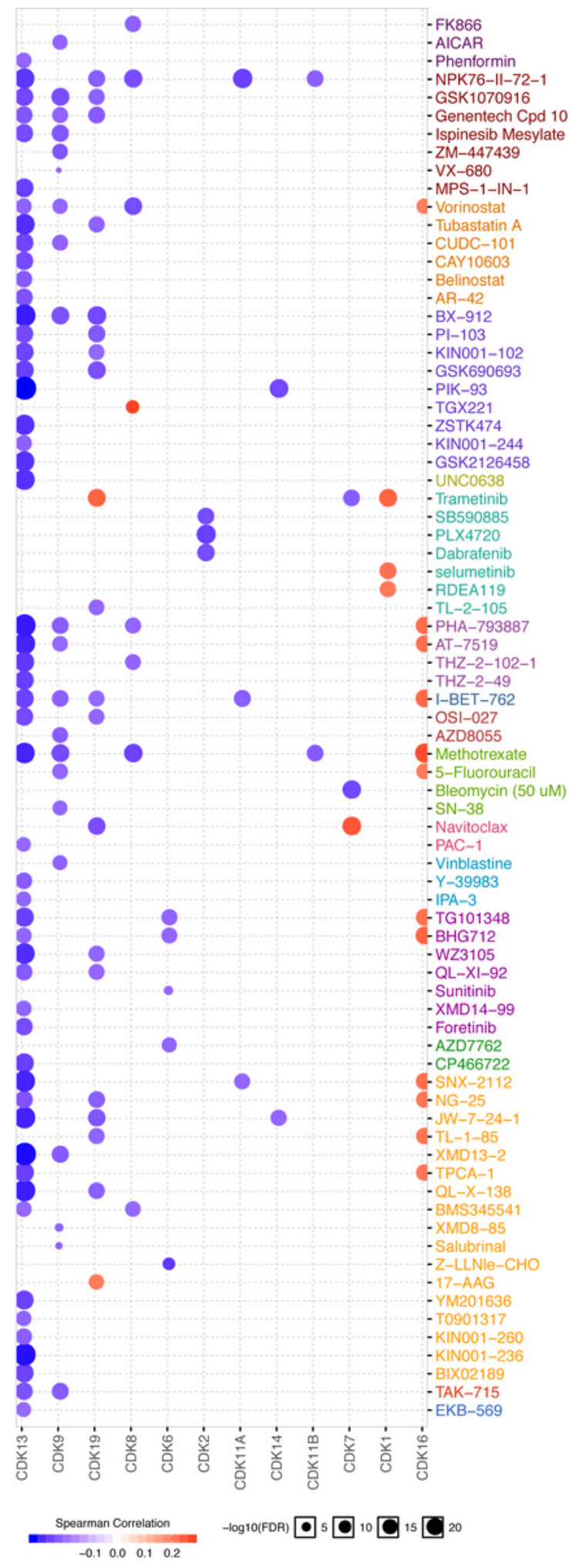

Figure 5

CDK family genes widely impact drug sensitivity in cancers. The dot represents the correlation between the CDK gene expression and drug sensitivity. The positive correlation represents that the high gene expression is resistant to the drug.

\section{Supplementary Files}


This is a list of supplementary files associated with this preprint. Click to download.

- Figures5.tif

- Figures2.tif

- Figures1.tif

- FigureS3.tif

- Figures4.tif 